\title{
CIENCIA Y TECNOLOGÍA EN LOS TELEDIARIOS COLOMBIANOS: SOBRE LO QUE SE CUBRE Y NO SE CUBRE ${ }^{1}$
}

RESUMEN: En este artículo, analizamos un corpus de noticias de ciencia y tecnología emitidas por el telediario Noticias Caracol - el de mayor rating en Colombia. Este trabajo atiende el doble objetivo de verificar si la demanda de los colombianos por este tipo de información es mínimamente atendida y de dar cuenta de las principales características de la cobertura de la ciencia y la tecnología en este telediario. Los resultados muestran que estos temas tienen una relativa poca relevancia en la agenda de este telediario y que los miembros del gobierno cumplen un papel preponderante, no sólo como voces, sino como fuentes en la construcción de los hechos noticiosos. También se encontró que este telediario no es necesariamente democrático a la hora de elegir sus fuentes, sus voces y sus referencias, pero además tampoco se dan elementos de contexto fuertes para estas informaciones.

Palabras clave: Noticias Caracol. Divulgación científica. Telediarios. Ciencia y tecnología. Análisis de contenido. Colombia.

\section{CIÊNCIA E TECNOLOGIA NOS TELEJORNAIS COLOMBIANOS: SOBRE 0 QUE SE COBRE E $O$ QUE NÃO SE COBRE}

RESUMO: Neste artigo, analisamos um corpus de notícias de ciência e tecnologia emitidas pelo telejornal Noticias Caracol - o com maior rating na Colômbia. Este trabalho atende ao duplo objetivo de verificar se a demanda dos colombianos por esse tipo de informação é minimamente atendida e de dar conta das principais características da cobertura de ciência e tecnologia nesse telejornal. Os resultados mostram que esses temas têm pouca relevância na agenda desse telejornal e que os membros do governo cumprem um papel preponderante, não somente como vozes, mas também como fontes na construção dos acontecimentos noticiosos. Também observamos que esse telejornal não é necessariamente democrático no momento de escolher suas fontes, suas vozes e suas referências, tampouco se dão elementos de contexto fortes para esse tipo de informações.

Palavras-chave: Notícias Caracol. Divulgação científica. Telejornais. Ciência e tecnologia. Análise de conteúdo. Colômbia.

DOI - http://dx.doi.org/10.1590/1983-211720175170110

\author{
Tania Arboleda Castrillón* \\ Daniel Hermelin** \\ Marina Ramalho*** \\ Luisa Massarani*** \\ Gabriela Reznik****
}

*Doctoranda en Ciencias Sociales y
Humanas de la Pontificia Universidad
Javeriana (Colombia)
Investigadora del Observatorio
Colombiano de Ciencia y Tecnología
-OCyT- (Bogotá)
Integrante del grupo de investigación
Sociedad, Ciencia y Tecnología en
Colombia del OCyT
Email: tarboleda@ocyt.org.co y
taniaarboleda@gmail.com
**agíster en Comunicación en la
Universidad de Borgoña (Francia),
Magíster en Enseñanza y Difusión
de las Ciencias y las Técnicas de la
Universidad de París XI (Francia).
Profesor del Departamento de
Humanidades y Jefe del Programa de
Comunicación Social de la Universidad
EAFIT (Medellín) y de esa universidad.
Integrante del grupo de investigación
Comunicación y Estudios Culturales del
mismo departamento.
Email: dhermeli@eafit.edu.co

***Doctora en el Área de Educación, Gestión y Difusión en Biociencias de la Universidad Federal de Río de Janeiro Periodista e investigadora del Núcleo de

Estudios en Divulgación Científica del

Museu da Vida, Casa de Oswaldo Cruz,

Fundación Oswaldo Cruz (Brasil)

Email: marina.fiocruz@gmail.com y marinar@coc.fiocruz.br

**** Doctora en el Área de Educación, Gestión y Difusión en Biociencias de la

Universidad Federal de Río de Janeiro

Investigadora del Núcleo de Estudios

en Divulgación Científica del Museu da

Vida, Casa de Oswaldo Cruz, Fundación Oswaldo Cruz (Brasil)

Investigadora Productividad de CNPq 1 C

Líder del grupo de investigación del CNPq Ciencia, Comunicación y Sociedad

E-mail: lumassa@fiocruz.br y luisa. massarani4@gmail.com

*****Especialista en Divulgación de la
Ciencia, la Tecnología y la Salud de la
Casa de Oswaldo Cruz/Fiocruz
Estudiante de maestría en el Programa
de Posgrado en Historia de las Ciencias
y de las Técnicas y Epistemología,
Universidad Federal de Rio de Janeirol
UFRJ (Brasil)
E-mail: gabrielareznik@fiocruz.br y

E-mail: gabrielareznik@fiocruz.br y
gabriela reznik@hotmail.com 


\section{SCIENCE AND TECHNOLOGY IN COLOMBIAN NEWSCASTS: ON WHAT IS COVERED AND NOT COVERED}

SUMMARY: In this paper we analyze a corpus of science and technology news broadcasted by Noticias Caracol - the TV news program with the highest level of audiences in Colombia. This article serves for the dual purpose of verifying whether the demand of Colombians for this type of information is minimally supplied and to account for the main features of the coverage of science and technology in this TV news. The results show that these issues had relatively low importance on the agenda of this newscast and government members play an important role, not only as sources but as voices in the construction of news stories. We also found that this TV news program is not necessarily democratic in choosing its sources, its voices and its references, and did not give strong elements of context for this kind of information.

Keywords: Noticias Caracol. Science communication. TV news. Science and technology. Content analisys. Colombia. 


\section{INTRODUCCIÓN}

En Colombia, la televisión ha sido el medio preferido por los colombianos para informarse, seguido de la radio y en tercer lugar la internet, cuyo consumo ha ido en crecimiento (AGUIRRE, 2005; OCyT-COLCIENCIAS, 2013). Según el Estudio General de Medios en Colombia ${ }^{2}$, los telediarios ${ }^{3}$ aparecen entre los primeros lugares de consumo en cuanto a los tipos de programa. Además, de acuerdo con la última encuesta nacional de percepción pública de la ciencia y la tecnología, la mayoría del consumo de televisión se divide entre los canales nacionales $(51 \%)$ y la televisión por suscripción (46\%). A la hora de informarse sobre ciencia, los colombianos prefieren este medio (62\%), seguido de internet $(30 \%)$. Con respecto a la tecnología, 53\% de la población encuestada utiliza televisión, seguido del 40\% que utiliza internet (OCyT-COLCIENCIAS, 2013).

Este panorama refleja el papel preponderante que ocupan los medios masivos de comunicación, y en particular la televisión, en la formación de las representaciones que en la actualidad los colombianos se hacen de la realidad en general, y de la ciencia y la tecnología en particular. Lo anterior no se aborda desde una perspectiva de audiencias pasivas, propia de "las trampas de las teorías de los efectos directos”, para usar la expresión de Maigret (2005), pero sí desde el punto de vista de la importancia que los medios tienen en nuestro contexto para poner a circular agendas sobre temas en general y sobre los relacionados con ciencia y tecnología en particular. Los medios contribuyen en la definición de las agendas ciudadanas, aunque no las garantizan, como se deduce entre diversos trabajos como el de Bonilla y Cadavid (2004), que, por lo demás, se ocupa de un objeto de estudio con medios y audiencias de Colombia, en que incluye ciencia y tecnología en las agendas.

Por su parte, Matiana González (2008) plantea que, desde una perspectiva habermasiana, los medios ponen sobre la mesa los asuntos sobre los que la sociedad ha de llegar a un consenso en cuanto a su significado se refiere y, al mismo tiempo, reflejan quiénes participan en las negociaciones, cumpliendo la función de un foro público en el que se dirimirían las distintas visiones que grupos sociales tienen sobre determinados objetos, incluido el de la ciencia y la tecnología. Sin embargo, siguiendo a Thompson (1995), esta autora a la vez nos advierte sobre la necesidad de no nos engañarnos con esta visión idílica, pues no todos los actores sociales tienen el mismo acceso a los canales de comunicación, y, aunque los receptores tienen la capacidad de intervenir en el proceso comunicativo, no hay que olvidar que se trata de un proceso asimétrico en donde la repartición del poder simbólico es siempre desigual (GONZÁLEZ, 2008). En estos procesos, los medios de comunicación no se limitan a informar. El hecho mismo de decidir a quién preguntan les da el poder de calificar como interlocutor válido a un grupo, descalificando como tales a cuantos otros opta por ignorar, como se desprende también de Martini (2000). 
Teniendo en cuenta el lugar privilegiado que ocupa la televisión en la vida de los colombianos y su intención de consumo de la ciencia y la tecnología a través de este medio, resulta relevante comprender tanto lo que los medios masivos en este contexto priorizan acerca de estas temáticas en la actualidad, como lo que dejan por fuera de ese complejo universo, en particular en lo que respecta a los telediarios. Sobre todo si tenemos en cuenta que ello está determinado en gran parte por lo que sus dueños, directores y editores consideran que las audiencias deberían saber, y que se constituye como la principal fuente que las audiencias de las sociedades occidentales han tenido para construir su idea de ciencia en las últimas décadas, como se discute en González (2008). Sin embargo, también habría que tener presente que la ciencia "se filtra" en las narrativas periodísticas, es decir que no todas sus representaciones son producto de decisiones intencionales, lo cual da cuenta de la profunda imbricación de este conocimiento en las sociedades que acogen los preceptos de la modernidad, y por lo tanto del carácter social de su construcción, como se analiza en Arboleda et al (2011) en relación con este asunto en Colombia.

Sin embargo, esta última afirmación también encuentra sustento en la discusión sobre el papel preponderante que cumplen los medios en el proceso creativo de la recepción frente a las temáticas de ciencia y tecnología, aunque el presente estudio no alcanza a abordar esta perspectiva - esto con base en abordajes que incluyan su conexión con las condiciones de producción y el análisis del mensaje, como se expone en Holliman (2004) y en Hermelin (2012) para temas de ciencia y tecnología. Una vez más, no podemos perder de vista los vínculos de los contenidos y las formas de enunciar con las demandas de las audiencias, la necesidad de plantear tales problemas en términos de las esferas públicas ampliadas con sus múltiples lógicas y racionalidades - allende de la visión habermasiana, para usar el concepto de Demers y Lavigne (2007) - y la inclusión de las preocupaciones y prioridades de las minorías.

Además, vale la pena vislumbrar el papel preponderante de las culturas populares y su relación con las formas de reconocimiento, en especial en lo que respecta a su relación con la ciencia y la tecnología en los medios en el ámbito colombiano y latinoamericano, como se propone en Hermelin (2013). Se trata de ver en las complejidades de la recepción las coincidencias de imaginarios de los medios y sus públicos, respecto a estos temas, para salir de la mirada de la omnipotencia de los primeros y la alienación de los segundos; una perspectiva a menudo atravesada por elitismos académicos que solo conciben esos procesos en una y sencilla sola vía. Cabe anotar que los estudios de recepción en relación con la ciencia y la tecnología son exiguos, como se observa en los balances de este sub-campo de los estudios de la comunicación, tanto en Colombia como en América Latina; así se muestra en Bonilla et al (2012) y en Jacks (2011), respectivamente.

Habiendo planteado estos asuntos de carácter teórico, la revisión de los pocos estudios empíricos realizados en Colombia indican una baja presencia de las temáticas de ciencia y tecnología en los medios, lo cual se evidencia en aspec- 
tos como: (1) la ubicación poco prioritaria que se le da a estos temas, ya sea en términos espaciales -primera página - o temporales - titulares de las emisiones -; (2) la predominancia del "unifuentismo", la falta de fuentes o la prevalencia de fuentes oficiales y en menor medida de académicos o especialistas; (3) la poca contextualización en términos de la relación que la ciencia y tecnología sostiene con diversos tópicos de la vida nacional, lo que puede estar asociado a que la mayoría de las informaciones se presentan en forma de resultados, con poca presencia de controversias y priorizando sucesos a nivel internacional - muchos de ellos provenientes de cables y de búsquedas en Internet -, entre otros. Estos trabajos se han concentrado en analizar la cobertura de estos temas en la prensa escrita, como se ve en Rozo (2006); en la aplicación de análisis de contenido a telediarios, como se ve en Arboleda et al (2011); o en analizar las agendas informativas generales de los medios masivos, entre cuyas temáticas se encuentran la ciencia y la tecnología, como aparece en Bonilla y Cadavid (2004) y en Gómez-Giraldo et al (2010).

Estos resultados no distan mucho de los encontrados en estudios sobre la cobertura de ciencia en la prensa escrita en otros países de América Latina (BUSTOS-MORA, 2004; PARODI; FERRARI, 2007; MASSARANI; BUYS, 2007; MASSARANI et al, 2008; ALMEDIA et al, 2011); estudios que, en algunos casos, se acercan a la concepción difusionista de la comunicación de la ciencia en el sentido de que plantean que los medios son incapaces de cumplir con esta tarea satisfactoriamente (siguiendo a BUCCHI, 2008). Si bien, otros análisis dan cuenta de dinámicas que ponen en evidencia que la presencia de la ciencia y la tecnología en los medios también depende de los científicos y académicos locales, cuando, por ejemplo, no responden a las solicitudes de información de los periodistas (AMORIM; MASSARANI, 2008). Hay que anotar que varios de estos trabajos han estado en parte influenciados teórica y metodológicamente por otros producidos en países considerados como "desarrollados", por ejemplo, el de Bauer et al (1993), y algunos de carácter comparativo entre países de la misma índole, como es el caso de Pellechia (1997) y de Peters et al (2008), que incluyen el tratamiento de este tipo de información en la prensa escrita y en otros medios.

En el presente artículo damos cuenta del análisis de un corpus de noticias de ciencia y tecnología emitidas por el telediario Noticias Caracol, el de mayor rating en Colombia, un lugar disputado con Noticias $R C N$, que pertenece al otro canal privado nacional de señal abierta en Colombia. Dichos noticieros compiten muy de cerca por ese primer lugar y en el momento en el que se decidió optar por este corpus, Noticias Caracol estaba, por poco, en el primer lugar, según el Estudio General de Medios antes mencionado. Este trabajo atiende el doble objetivo de verificar si la demanda de los colombianos por este tipo de información es mínimamente atendida - al menos la que se advierte en estudios de percepción en el país como los ya citados -; y de dar cuenta de las principales características de la cobertura de la ciencia y la tecnología en los telediarios, con una mirada crítica del periodismo televisivo sobre estos temas - en una dirección similar a lo que se desprende del estudio descrito en Massarani y Ramalho (2012) para telediarios 
latinoamericanos, que también incluyó un análisis del telediario más visto en Brasil (RAMALHO; POLINO; MASSARANI, 2012). Además, se busca ampliar el panorama de análisis para contribuir a la comprensión de las maneras complejas mediante las cuales la ciencia es puesta al alcance de los públicos en el contexto colombiano y el modelo de ciencia que lleva implícita dicha manera de presentarla, como se plantea en un estudio como el de González (2008).

Un conjunto de preguntas se desprende desde esta perspectiva, entre otras: (1) ¿qué modos de comunicación de la ciencia y la tecnología tiene el telediario y a qué intereses responde, en aras de influenciar las agendas de los ciudadanos?; (2) ¿es posible determinar, mediante el análisis de contenido de las noticias, cuáles son los objetivos del telediario, sus escalas de valores e ideología frente este tipo de conocimiento y sus aplicaciones?; (3) ¿qué grupos sociales tienen cabida para transmitir a la audiencia su visión particular de la ciencia y la tecnología? A continuación, intentaremos responder preguntas de este tipo. En términos más generales, vamos a acercarnos a cómo se construyen las representaciones mediáticas de la ciencia y la tecnología basados en uno de los dos telediarios colombianos de mayor audiencia, y a su posible eco en las representaciones sociales de sus públicos.

\section{ASPECTOS METODOLÓGICOS}

Para este análisis, se constituyó un corpus a partir de una muestra de un año de emisiones del telediario Noticias Caracol, entre abril de 2009 y marzo de 2010, que fue recogida mediante grabaciones de las emisiones en vivo. Un conjunto mínimo de estas emisiones no pudo ser registrado o presentaba deficiencias, por lo tanto fueron solicitadas y autorizada su utilización por la dirección del archivo audiovisual de la cadena Caracol Televisión, teniendo en cuenta que su utilización era para fines de investigación sin ánimo de lucro. La selección se hizo a partir de la metodología de semana construida, con base en trabajos clásicos como el de Krippendorf (1990), y en aplicaciones recientes para temas cercanos al nuestro como las que aparecen en los estudios mencionados en América Latina. En total son 72 emisiones las analizadas, es decir, seis emisiones semanales durante doce meses. No se tuvieron en cuenta los domingos, con fines de hacer un trabajo comparativo con otros países de América Latina - en especial con el telediario brasilero Jornal Nacional (del emporio mediático brasilero Globo), cuyo estudio aparece en Ramalho, Polino y Massarani (2012). Es importante aclarar que, durante 2012, se presentaron algunos cambios relevantes de forma y de fondo en la emisión de Noticias Caracol, debido a un cambio de dirección del telediario.

Nuestra herramienta de análisis de contenido aparece explicada en detalle en Ramalho et al (2012), y fue producto del trabajo colaborativo desarrollado en el marco de la Rede Ibero-americana de Monitoramento e Capacitação em Jornalismo Científico ${ }^{4}$. Para seleccionar cada unidad noticiosa de análisis sobre ciencia 
y tecnología, usamos el mismo criterio de Ramalho, Polino y Massarani (2012) desarrollado a partir de Rondeli (2004), que indica que para ser incluidas en la muestra, las notas deben cumplir con uno o más de los siguientes criterios: mencionar científicos, investigadores académicos o profesionales vinculados a instituciones científicas, siempre y cuando éstos traten temas científicos en la nota; usar datos científicos ligados a resultados de investigaciones; hablar de política científica; o de divulgación científica.

Las variables del protocolo de análisis de contenido para las notas de ciencia en el telediario a las que les dimos prioridad son: la cantidad total y relativa de notas; la duración; la aparición en los avances (o titulares: esta es la expresión que se emplea en Colombia); la aparición según el bloque de noticias; las áreas del conocimiento; los frames para analizar algunos elementos narrativos; los recursos visuales; las informaciones de contexto; las controversias; la mención de beneficios, promesas, daños o riesgos de la ciencia; la aclaración de términos científicos; las recomendaciones; las fuentes y las voces; el origen geográfico de los científicos y de los hechos noticiosos; y el género de los científicos entrevistados.

A pesar de la fortaleza de la taxonomía de dicho protocolo, no se agota la discusión sobre la posibilidad de que algunas variables se mezclen, en especial con los frames $^{5}$; o se puedan tener en cuenta en el diseño de otros. Al fin y al cabo, el protocolo que usamos es una herramienta sólida de análisis, pero que, por supuesto no está cerrada a los ajustes, ni adaptaciones para estudios futuros de naturaleza similar.

Vale la pena señalar también que en un trabajo anterior, con un corpus cercano al de este artículo (ARBOLEDA et al, 2011), y que se basa en nuestro protocolo, se usaron los criterios para la selección de las notas pero se permitió incluir unos cuantos temas, independiente de que cumplieran con los mismos: por ejemplo, notas de cambio climático, gripe H1N1, biodiversidad, recursos hídricos, entre otros. Además, el corpus allí tratado está constituido por los últimos seis meses del presente estudio -entre octubre de 2009 y marzo de 2010. Esto hace que muchos de los resultados no sean comparables con otros estudios que usan el protocolo. Sin embargo, allí se discuten algunas perspectivas para combinar el trabajo de este tipo de análisis de contenido con herramientas propias del análisis semio-discursivo y pragmático -pragmático en el sentido del campo de estudio cercano a la filosofía del lenguaje como se puede ver en Escandell (1999) -, como un complemento que permita fortalecer la aproximación al objeto de estudio y que, en combinación con enfoques sobre el lugar de las condiciones de producción, permita conexiones con los estudios de recepción cuantitativos y cualitativos, a manera de una suerte de visión integral sobre el circuito comunicativo en relación con los temas de ciencia y tecnología, como se arguye en Hermelin (2012) ${ }^{6}$. 


\section{RESULTADOS Y ANÁLISIS DE RESULTADOS}

En las 72 emisiones analizadas de Noticias Caracol - obtenidas mediante la metodología de semana construida aleatoriamente -, encontramos un total de 38 notas sobre ciencia y tecnología que cumplen los criterios que expusimos. Esto quiere decir que hay un promedio de 0,53 notas por emisión, que no es muy alto si lo comparamos con lo encontrado en el estudio similar en Brasil, realizado por Ramalho, Polino y Massarani (2012), en el que el promedio fue de una nota por emisión. Además, si se tiene en cuenta que algunas de las emisiones de Noticias Caracol duran alrededor de 60 minutos, aun considerando que esta duración incluye los tiempos de publicidad, el número sigue siendo relativamente bajo.

A este respecto, llama la atención también la duración total de las notas: 29 de ellas (el 76,3\%) tardan entre 0 y 2 minutos, de las cuales 9 tardan entre 1:31 minutos y 2 minutos. Las otras 9 notas duran entre 2:01 minutos y 3:31 minutos. Es decir, no se trata de notas particularmente largas, máxime si se tiene en cuenta su índole: las notas de ciencia y tecnología que cumplan con los criterios de cientificidad para poder ser incluidas en nuestro análisis son notas que, a priori, el público no naturaliza tan fácil como otros temas y, por lo tanto, se podría esperar que requieran un nivel de contextualización más alto que el promedio de otros temas noticiosos, lo que se traduciría en una mayor duración de las mismas. Esto se podría deducir del hecho de que, siendo que en promedio se emite cerca de media nota $(0,53)$ en cada emisión, y que por lo tanto es un tipo de nota poco común en este telediario. No obstante, esto hay que confrontarlo con otros elementos que se verán luego, e incluso tras hacerlo, será difícil ir más allá de una afirmación hipotética. También es importante mirar nuestro relativo bajo número de notas que cumplan con tales criterios sin caer en sesgos deficitarios, de los que es difícil desprenderse a veces en la investigación y en las prácticas de comunicación de la ciencia, como lo señalan Daza y Arboleda (2007). Vale la pena aclarar que en las visiones deficitarias de la comunicación de la ciencia y la tecnología se parte de la base de que es bueno per se difundirlas, pues esto sirve para tener una sociedad que valide y se apropie de las bondades de las mismas - varias discusiones amplias sobre este punto aparecen en Pérez-Bustos y Lozano-Borda (2011).

Ahora bien, 11 noticias tienen avance o están en los titulares (o avances) en la apertura del telediario, lo que representa el $28,9 \%$ del corpus ${ }^{7}$, de lo cual se deduce que no hay mayor preponderancia de las noticias de ciencia frente a otras como las de conflicto armado y "violencia común", ni frente a las de carácter de agenda política, por ejemplo ${ }^{8}$.

Por su parte, la aparición de estas notas se da sobre todo en el segundo bloque, con un total de 18, es decir, el 47,3\%; los otros dos bloques, el primero y el tercero, contienen 10 notas cada uno $(26,3 \%)$. Con esto constatamos que el telediario le otorgaría una importancia media (moderada) a este tipo de noticias, si se considera que el primer bloque es el de mayor importancia noticiosa. Pero a partir del bloque 3, las noticias se concentran en deportes y en la sección de 
farándula - lo que a veces incluye un bloque 4, o incluso da para bloques posteriores, con los anuncios publicitarios. Hay que agregar que en este telediario, como en otros de alto rating en Colombia, aparece cierto grado de irregularidad en el manejo de temas y secciones, como se muestra en Gómez-Giraldo et al (2010). Salvo las dos secciones mencionadas, la de noticias internacionales, la de datos económicos, y la de una suerte de notas "confidenciales" de política (que comúnmente se combinan con datos sin confirmar e incluso rumores, desde hace un tiempo llamada El Código Caracol), y una sección de recorridos por zonas del país (patrocinada en la época del corpus por Bancolombia - uno de los bancos más grandes y de mayores utilidades de Colombia -, y en la que aparecen algunas notas que clasificaron para entrar en nuestro corpus), los demás temas no están por lo general agrupados; menos aún en el caso de los noticias sobre ciencia y tecnología.

En cuanto a los días de la semana, aquellos con mayor concentración de notas son los miércoles y los viernes, con 9 notas cada día, y los lunes con 7; allí se concentra el 65,7\% (25 notas). Los martes y sábados el número de notas desciende a 6 y el día con el menor número es el jueves, con 1 . Se podría esperar que aumente la tendencia con este tipo de notas acercándose el fin de semana, dados fenómenos como que la agenda política tiende a bajar. No obstante, los resultados son aleatorios ${ }^{10}$. En lo que concierne a la distribución de notas por meses, se encontró una mayor concentración de notas en febrero de 2010 (26,3\%); le siguen julio de 2009 (15,8\%), y marzo y diciembre de 2009 $(13,1 \%$, cada uno). Esto contrasta con agosto, que no presenta ninguna nota. Es difícil, pues, pensar en una decisión editorial para entender esto; se podría lanzar una hipótesis para trabajos futuros: julio y febrero son los meses del retorno escolar, y entre los dos suman más del 40\%. Pero esto requeriría un acercamiento al lugar de las condiciones de producción, en el sentido de Hermelin (2012) ${ }^{11}$.

En suma, si bien de variables como los días de la semana y las notas por mes no obtuvimos tanta información, no pasa lo mismo con las demás variables. De allí se puede deducir que en lo que respecta a la relevancia en la agenda, el tipo de notas de nuestro corpus está lejos de ser una prioridad: no son notas de valores altos (a veces incluso exiguos) en cuanto a frecuencia, a duración, a los avances y a la aparición según el bloque. Aunque esto se podría contrastar con otros tipos de estudios como los de agenda setting -y una referencia clásica útil para esto es McCombs y Shaw (1993)-, vuelve una inquietud sobre lo planteado antes: es claro que en este telediario colombiano no es el tipo de información con más relevancia, pero hay notas que tal vez toquen temas de ciencia y tecnología de forma transversal, como se muestra en Arboleda et al (2011); y esto haría pensar en las posibilidades de ajustes para el protocolo, según los países. Pero esto le quitaría fuerza como herramienta para trabajos comparativos entre los mismos, como se expresa en Ramalho et al (2012). 
Figura 1: distribución de las notas por áreas de conocimiento

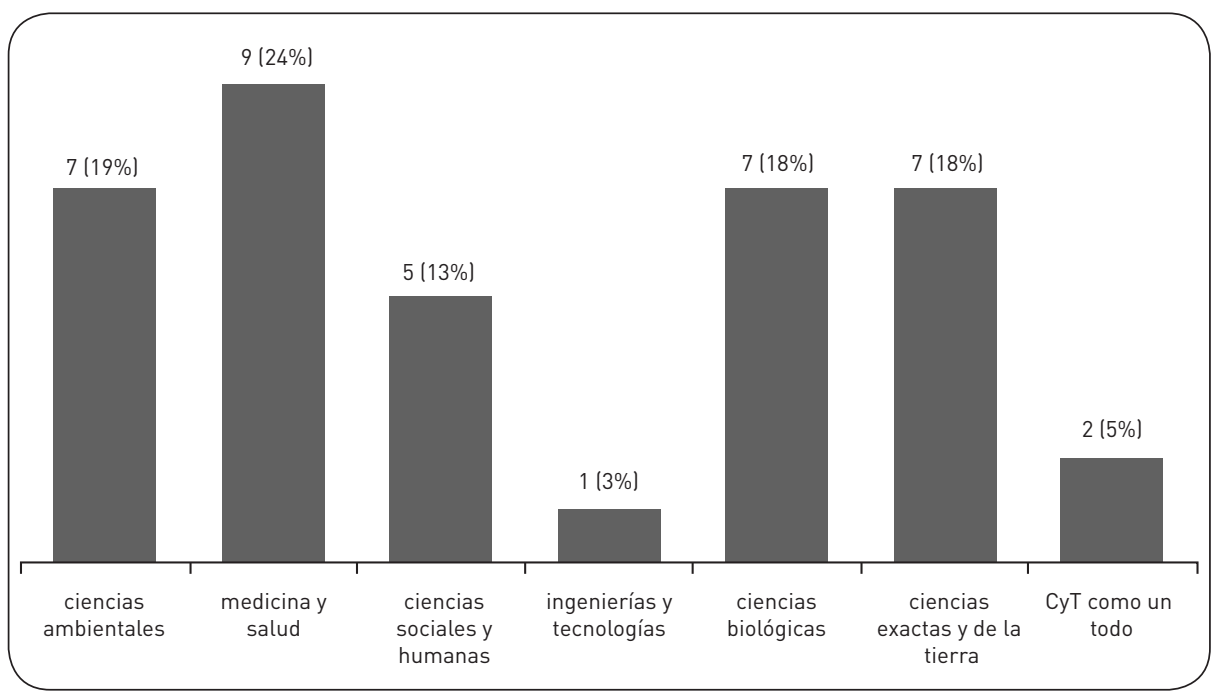

Por otro lado, hay una variable clave en este estudio que tiene que ver con la distribución por área del conocimiento - su disección se basa en los criterios de Ramalho et al (2012), y sus resultados se observan en la figura 1. Se destaca lo siguiente: el 24\% (9 notas) de las notas son sobre medicina y salud, de las cuales 3 tienen que ver con el virus H1N1 (su propagación, real y potencial, fue una coyuntura noticiosa significativa, especialmente entre abril y junio, por supuesto no sólo en Colombia). Las siguientes áreas tienen el mismo número de notas (7), que representan el 18\% del corpus cada una: ciencias exactas y de la tierra, ciencias biológicas y ciencias ambientales. El área de ciencias sociales y humanidades se vio reflejada como área principal en 5 notas $(13 \%)$. Las áreas de menor visibilidad son CyT como un todo con 2 notas $(5 \%)$, las ingenierías y tecnologías con 1 nota $(3 \%)$, y ciencias agrarias que no aparece representada en las noticias del corpus, según esta categorización para escoger la principal área del conocimiento. Vale la pena subrayar que sólo 3 notas, todas de medicina y salud, estuvieron en el primer bloque, e igual número en los avances o titulares.

En el caso del primer lugar de medicina y salud es algo que no es nuevo en este tipo de estudios en contextos más o menos cercanos, como se puede ver en Arboleda et al (2011), Ramalho, Polino y Massarani (2012), y Almeida et al (2011). En el de Ramalho, Polino y Massarani (2012) se citan estudios en contextos diversos, algunos de países desarrollados, donde se encuentra esta misma tendencia. Además, si esto se contrasta con que la mayoría de estudios de recepción en América Latina, relacionados con temas de ciencia y tecnología, están ligados a la salud (y muchos también al medio ambiente), como se observa en el balance de Jacks (2011), el resultado no es sorprendente. Al fin 
y al cabo, se trata de temas en los que las personas se sienten más representadas individual y colectivamente; y donde su alta demanda, en especial en el público colombiano - como lo muestran Bonilla y Cadavid (2004) -, parece ser atendida por el telediario (esto para no hablar del rol creciente que un medio como éste viene teniendo en el manejo de temas de salud en Colombia. Esto como en una suerte de "gracias a nosotros, tenga un médico en su casa". Sin embargo, según nuestro protocolo de análisis, no incluimos noticias de salud si no cumplían con las restricciones de cientificidad que se señalaron).

Aunque con menos fuerza, ciencias ambientales ocupa el segundo lugar, compartido con ciencias biológicas, ciencias exactas y de la tierra. En el primer caso, como se analiza en Arboleda et al (2011) y en Hermelin (2012), se puede ver también como una respuesta a agendas con las que los públicos se sienten más reconocidos: los temas ambientales suelen estar relacionados con asuntos que afectan la cotidianidad de las personas. Aquí también hay que agregar que 3 de las notas tomadas como ciencias ambientales están ligadas a la cumbre mundial sobre cambio climático realizada en Copenhague, en diciembre de 2009. Se trata de notas que además estuvieron relacionadas con política científica.

Por su parte, las otras dos áreas con las que se comparte el segundo lugar son áreas en las que algunas notas pueden tener cercanía con las ciencias ambientales; es el caso de las notas sobre biodiversidad y geología: allí hay aspectos que pueden estar ligados. Es necesario anotar lo que se señala en Arboleda et al (2011) y Ramalho et al (2012), en relación con la dificultad para determinar la principal área de conocimiento en cada noticia: en varios casos en las noticias se podrían identificar varias áreas al tiempo, si bien se prioriza la que es más apropiada para el tema central de la nota. Esto obliga al codificador a excluir otras, por lo que no se estaría reflejando la complejidad de los procesos actuales de producción y aplicación del conocimiento que son inter o transdisciplinarios, con frecuencia organizados por problemas coyunturales que hay que atender. Esta dificultad para determinar claramente las áreas de conocimiento nos puede decir mucho de la producción de sentido que involucra contenidos científicos en los formatos informativos televisivos, en este caso específico del contexto colombiano. Al menos así parece desprenderse de esta muestra, en la que claramente no hay un área manifiestamente preponderante. Esto contrasta con Ramalho, Polino y Massarani (2012): allí se señala que en el telediario de Brasil, medicina y salud son claramente preponderantes. 
Figura 2: frames (o encuadres) utilizados en las notas

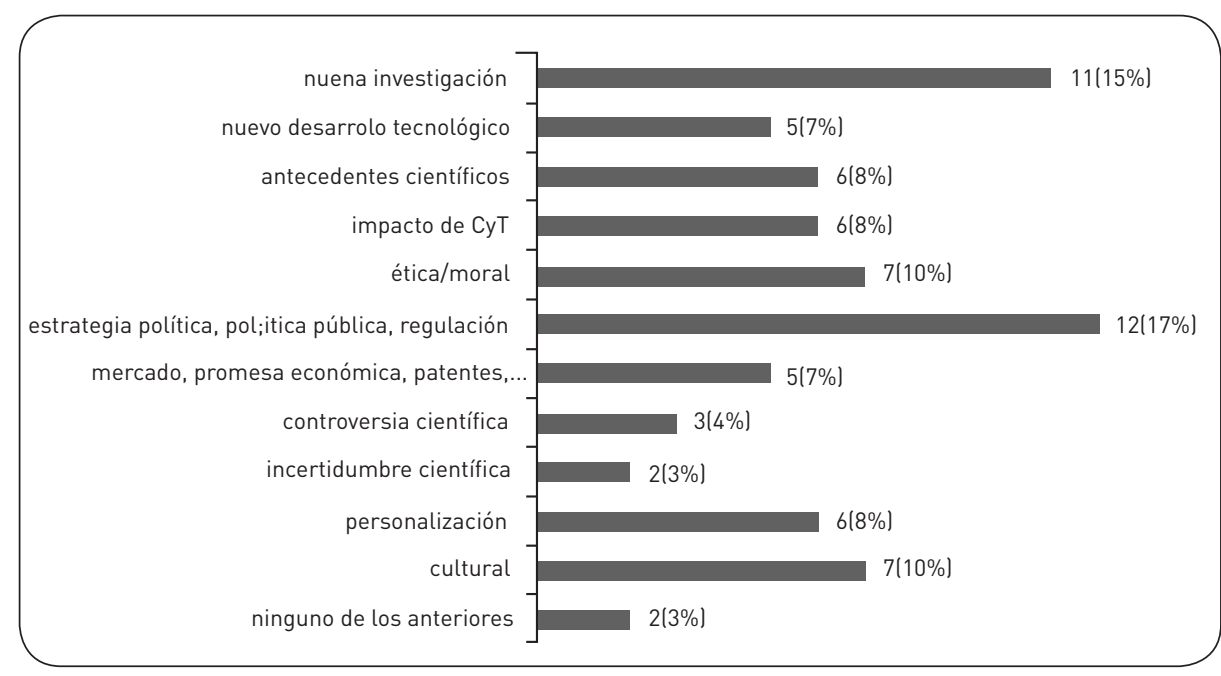

En lo que respecta a los frames (o encuadres), vamos a detenernos en algunos de los datos cuya clasificación aparece en la figura 2. En el protocolo que usamos, una nota podía ser clasificada hasta con tres tipos de frames diferentes (por eso el número total de frames es bastante superior al número total de notas). De las 38 notas, 12 (casi el 38\%) presentan el encuadre "estrategia política, política pública, regulación”, con lo cual se puede inferir que explicitan una estrecha relación entre ciencia y decisiones del ámbito gubernamental o normativo, ya sea a nivel nacional o internacional. Las ya bastante demostradas relaciones estrechas entre ciencia y poder tienen su expresión en este caso.

Paralelamente, en las noticias se encontró una muy baja presencia de los encuadres "controversia" e "incertidumbre" que, en principio, podrían "compensar" de alguna manera esta tendencia, en tanto muestran la ciencia como un proceso de producción de conocimiento que no se da por hecho, y que está en constante definición. En términos del primero, hay 3 (de los cuales 2 se refieren a un mismo hecho que se relata en varias noticias ${ }^{12}$ ) y en el segundo hay 1. Esto puede sugerir una baja representación de la ciencia en el telediario como un proceso en construcción, no exenta de dificultades a la hora de establecer regímenes de verdad - como se discute en varios de los trabajos que aparecen en Pérez-Bustos y Lozano-Borda (2011). No obstante, lo anterior tiene un contraste, aunque no muy fuerte: las 7 notas del frame "ética/moral", y las 6 notas que arroja el frame "impacto de CyT", dado que ambos encuadres pueden incluir, aunque a menudo de manera tácita, narrativas de controversia.

Mientras tanto el encuadre "nueva investigación" se evidencia en 11 notas $(37 \%)$, valor cercano al de mayor presencia, que es como ya mencionamos el de "estrategia política". Se habría podido esperar, dada la naturaleza 
del protocolo, un resultado más alto en dicho encuadre en tanto éste le da bastante importancia a seleccionar las notas que estén relacionadas con resultados, personas e instituciones ligadas a la investigación científica. Lo anterior también se debe mirar a la luz de que sólo 6 notas $(16 \%)$ tuvieron el encuadre "antecedentes científicos". Esto, por lo demás, es interesante confrontar con los resultados del análisis cuantitativo de la agenda, en relación con el bajo número de notas de nuestro corpus, y su relativa baja relevancia, como se mostró al principio de nuestro análisis de resultados.

Como se dijo, hay otras variables que en el protocolo fueron consideradas como variables de tratamiento de las notas. En algunos casos estas podrían interrelacionarse con algunas propias de los frames, pues tienen un vínculo significativo con las dimensiones narrativas. Vamos a destacar los resultados de algunas de ellas. En primer lugar está el manejo de las imágenes. De las 38 noticias, $15(39 \%)$ no utilizaron los recursos visuales analizados (imagen de científico ; tabla de datos, infografías o diagramas; animación $\left.{ }^{14}\right)$, y 23 (61\%) sí lo hicieron de las siguientes formas: 17 noticias utilizaron un recurso visual, de ellas 12 presentan la imagen de un científico, 3 notas hicieron uso de tablas, infografías o diagramas, y 2 de animaciones; 5 noticias tienen dos recursos visuales: una noticia contiene animación e imagen del científico y en 4 apelaron a tablas de datos, infografías o diagramas además de la imagen del científico. Una noticia contiene los tres recursos visuales: tablas, animación e imagen del científico. Los científicos están presentes visualmente en 18 noticias (47\% de las notas del corpus), en 12 (32\%) de éstas aparece como único recurso visual Este manejo de imágenes se puede contrastar con una variable que fue significativa en el protocolo: 23 de las 38 noticias presentaron alguna información de contexto -aunque el protocolo no pide especificar en qué grado - que relaciona el acontecimiento con otros recientes, con antecedentes históricos u otros aspectos para enmarcar el hecho noticioso (lo que equivale al 61\%, curiosamente el mismo porcentaje de noticias que usan recursos visuales; pero no coinciden las mismas notas). Esto contrasta con el resultado de otras dos variables: en 13 (34\%) notas aparece una aclaración de términos científicos especializados. De las 38 noticias del corpus, 10 (26,3\%) presentan controversias de diverso tipo; de esas, sólo 3 presentan controversias de carácter científico ${ }^{16}$. Esto da fuertes indicios en relación con la intención de un contrato de lectura con el público en el que se intenta mantener un lazo de estabilidad con el mismo; un contrato que se apoye, sino en la veracidad de la noticia, sí en su verosimilitud, para usar los conceptos de Martini (2000) - ᄀy esto dialoga con algo de lo señalado en los frames sobre los regímenes de verdad. Es decir, se puede caer en lo que Moirand (1997) llama efecto de didacticidad: la intención real o simulada por parte de los medios de volver al público más competente; en este sentido, lo legible o visible puede reemplazar a lo comprensible o inteligible. Se puede considerar que el telediario da algunos bandazos en esta dirección, sin que con esto queramos hacer de ventrílocuos del público y sus procesos de recepción real, como ya se advirtió en la introducción de este trabajo. 
Lo anterior no se puede desligar de variables ligadas al tratamiento de las notas en términos de beneficios, promesas, daños o riesgos de la ciencia; 20 de las notas hablan de estos temas en términos de beneficios y en 17 hablan en términos de promesas. En contraste, 9 de las notas hablan en términos de daños y 15 en términos de riesgos. Es decir, se puede considerar que, en promedio, $58 \%$ de las notas hablan en términos positivos (o beneficios o promesas o ambas) de la ciencia; y que, en promedio, 32\% de las notas hablan en términos negativos (de daños, riesgos o ambas) de la ciencia. Esta tendencia es algo que también aparece en un trabajo como el de Ramalho, Polino y Massarani (2012), para el caso brasilero - y que, cabe señalar, se asemeja a los resultados de percepción pública de la CyT en Colombia, en OCyT - Colciencias (2012). Además, 13 (34\%) de ellas hablan en términos de recomendaciones de la ciencia al telespectador. No predomina entonces una visión en el telediario sobre la ciencia y la tecnología, propia de lo que Beck (2005) denomina las sociedades del riesgo, y su anclaje al problema individual y colectivo del control de incertidumbre, que implica la modernidad frente a la pérdida de lazos de estabilidad, las formas de control y los desafíos de la autonomía tan común en las sociedades "desarrolladas", como lo expone Le Breton (1995) ${ }^{17}$.

En lo que respecta a los resultados de las fuentes y las voces utilizadas por el telediario en este tipo de hechos noticiosos, es importante tener en cuenta que una nota puede tener más de una fuente y más de una voz. Y toda voz constituye una fuente, pero no al revés. Aquí vale la pena resaltar lo siguiente: la mayoría de las fuentes están en el ítem "científicos, profesores universitarios, investigadores, instituciones de investigación”, pues están presentes en el 63\% del corpus, y representan el 32\% del total de tipos de fuentes. Le siguen: "miembros del gobierno": $22 \%$ del total de tipos de fuentes; "expertos o profesionales especializados (no vinculados a ninguna institución)": 14\% del total; "ciudadanos y miembros del público": 7\% del total; y "representantes industria, comercio y productores": 7\% del total. Se ve pues una importancia relativa muy fuerte por parte de las fuentes del gobierno en contraste con las de los "más autorizados" (esto, si lo asumimos de desde una mirada cientifista, o desde una perspectiva difusionista de la ciencia). Esto se puede confrontar con datos anteriores como los que arrojó el frame "estrategia política, política pública, regulación”, que dio los resultados más altos. Por lo demás es importante anotar que estos datos son bastante diferentes del estudio similar de Ramalho, Polino y Massarani (2012) en Brasil, donde sólo la fuente “científicos” está presente en el $81 \%$ del corpus. 
Figura 3: números totales de tipos de voces presentes en las notas

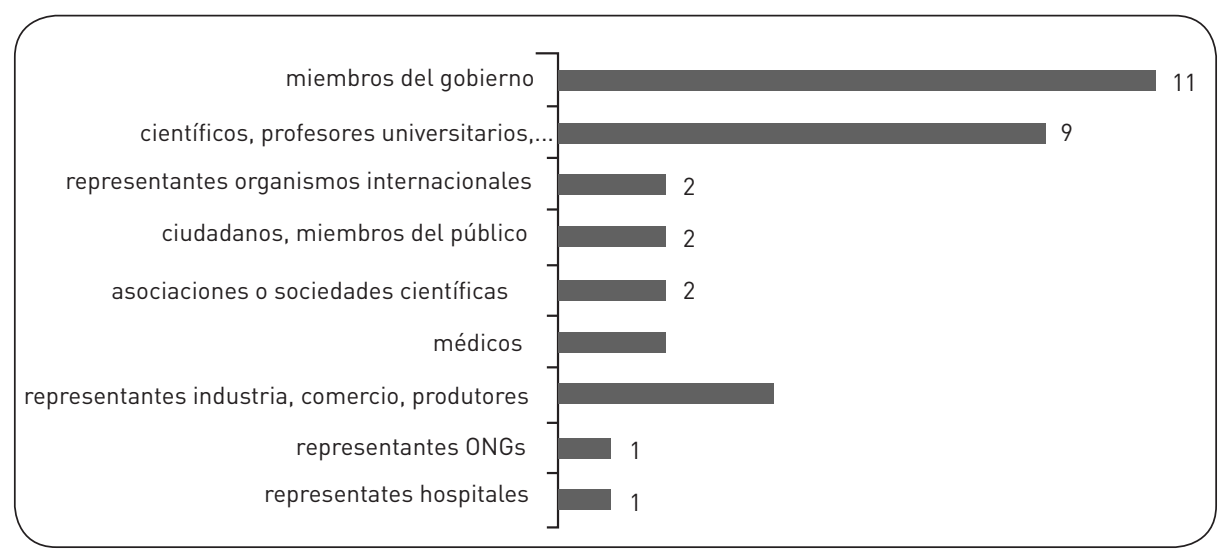

Sobre las voces se destaca lo siguiente (los datos absolutos se pueden observar en la figura 3): representativamente, tienen más voz las fuentes gubernamentales que las científicas puesto que de 16 notas que tienen como fuente al gobierno $^{18}, 11$ emiten su voz ( $32 \%$ del total de tipos de voces); mientras que de 24 notas que tienen como fuente a los científicos o sus instituciones, 9 emiten su voz ( $26 \%$ del total de tipos de voces). De estas últimas, 4 son representantes de instituciones del gobierno: esto se da porque hay varias instituciones que en Colombia hacen investigación pero que a su vez son instituciones gubernamentales, como es el caso del Ingeominas, IDEAM y Corantioquia, que además de producir conocimiento científico, cumplen funciones de regulación y gestión de la política pública en temas concretos ${ }^{19}$.

¿Será posible aventurarse a decir que en el telediario la representación de la ciencia como empresa de producción de conocimiento independiente no parece ser una constante en el ámbito colombiano? Si asumimos que la fuente científica representa una autoridad epistémica que produce verdades, tenemos que el $22 \%$ de las noticias en las que el gobierno aparece como fuente, lo hace apelando al argumento científico que cumple una función de autoridad epistémica para validar posturas y construir la realidad, además de la situación de poder en la que ya los miembros del gobierno se encuentran perse. De una u otra manera, es clara la preponderancia de este actor en la construcción de las noticias sobre ciencia y tecnología en nuestro corpus.

Otras variables que merecen atención son la aparición de científicos según el género (en este caso los entrevistados, es decir, que aparecen como voces), según su origen geográfico y según el lugar del evento noticioso. En cuanto al género, el 84\% de los actores científicos que aparecen son hombres, y el 16\% son mujeres. Esto no "se compadece" con la realidad de los actores científicos en Colombia donde alrededor del $40 \%$ de científicas activas son mujeres ${ }^{20}$. Parece pues una visión sesgada del telediario, que podría connotar una suerte de estereotipo 
machista para las voces de carácter científico. Por supuesto, esto requeriría de una aproximación más cuidadosa, en especial, como se dijo antes, en su relación con el lugar de las condiciones de producción, en combinación con estudios de género en esta dirección.

En lo que concierne al origen geográfico de los científicos o de las instituciones de investigación que generan los hechos noticiosos, los resultados muestran que el 50\% de las notas mencionan a científicos o instituciones de investigación del propio país -Colombia en este caso -; en 20\% de las notas, estos actores son de Europa; en 13\% son de América del Norte; y en 10\% son de América Latina - excluyendo a Colombia. Esto contrasta con la localización del evento u objeto de investigación de que trata la nota: en 55\% de las noticias, los eventos son nacionales; 18\% son en América del Norte; 13\% en Europa; 3\% en América Latina. (El protocolo se basa en categorías como "países desarrollados fuera de Europa y de América del Norte", y "otros países en desarrollo fuera de América Latina". No dejan, sin embargo, de ser categorías difíciles de estabilizar si se miran a la luz del auge económico de las potencias emergentes como los denominados BRICS (Brasil, Rusia, India, China y Sudáfrica).

A grandes rasgos, se puede decir que hay una suerte de "dominio de la ciencia nacional" en el telediario. Sin embargo llama la atención las cifras de Europa y de Estados Unidos en comparación con las de América Latina: en la localización de los eventos son mucho mayores; también en el origen geográfico de los científicos (o las instituciones que dieron lugar al hecho noticioso). Esto nos habla de una cierta vigencia del centrismo anglo-europeo en las agendas de los medios, al menos en la de Noticias Caracol. Algo que es difícil de desconectar de la realidad del avance de las prácticas y de la investigación en nuestros países del Sur, en el subcampo de la comunicación pública de la ciencia y la tecnología, como lo discute Hermelin (2011).

\section{CONCLUSIONES Y PERSPECTIVAS}

Los resultados que arroja nuestro análisis nos muestran que los temas de ciencia y tecnología tienen una relativa poca relevancia en la agenda de uno de los dos telediarios con más alta audiencia en Colombia, Noticias Caracol. Esto se ve desde el punto de vista cuantitativo, pero también cualitativo. El protocolo que utilizamos nos es muy útil para detectar esto. Sin embargo, no siempre nos permite ver maneras de abordar la mediatización de la ciencia y la tecnología que no estén ancladas explícitamente a la institucionalidad científica y a los resultados de investigación, y a su relación con políticas científicas y prácticas de divulgación científicas de manera manifiesta. Aparece entonces la necesidad de plantear nuevas perspectivas en este sentido que puedan, tal vez, incluir expresiones más transversales de estas temáticas, al menos para el ámbito colombiano. Ahora bien, no todo lo que tiene que ver con la ciencia 
y la tecnología está ligado a los cánones de autoridad científica o estatal. Las encuestas de percepción, y en especial las realizadas en Colombia, dan pautas en esta dirección: los públicos se acercan, se apropian e incluso proponen asuntos relacionados con la ciencia y la tecnología que en ocasiones responden a dichas miradas transversales. Pero es difícil, para poder estudiar estos temas, desprendernos de un cierto difusionismo científico y de ciertas herencias del centrismo anglo-europeo: al fin y al cabo se puede caer en el problema de que todo abordaje del conocimiento sea considerado como científico.

Sin perder de vista lo anterior, es importante resaltar el papel que, en el caso colombiano, tienen los miembros del gobierno, no sólo como voces sino como fuentes en la construcción de los hechos noticiosos. Y lo tienen no sólo en lo que respecta a políticas relacionadas con ciencia y tecnología, sino con muy diversos acontecimientos científico-tecnológicos. Esto parece indicar que si nos quedásemos esperando una óptica más difusionista por parte de los medios colombianos - o al menos de este telediario - para presentar este tipo de temas, las batallas contra los poderes políticos, y en especial gubernamentales, parecen perdidas, al menos parcialmente. Lo mismo se vislumbra en cuanto a la autoridad y representatividad que les confieren a los múltiples actores: este telediario no es necesariamente democrático a la hora de elegir sus fuentes, sus voces y sus referencias (como sucede con las fuentes de científicas mujeres, y con las voces de la población). Pero además, no hay tampoco una tendencia marcada a dar elementos de contexto fuertes para informaciones de esta índole, que los podrían requerir en aras de una mayor apropiación social de los mismos.

Ahora bien, un trabajo como este vale la pena que se confronte con estudios de otros medios similares en el propio país y en otros. Y también con otros medios no sólo los masivos tradicionales, sino con el auge de los usos sociales de las "nuevas" TIC. Al fin y al cabo allí se dan tantos elementos para el empoderamiento de la población como para su control. Y en esto la ciencia y la tecnología juegan un papel fundamental.

\section{NOTAS}

${ }^{1}$ Este artículo hace parte de un proyecto desarrollado entre el Departamento de Comunicación de la Facultad de Comunicación y Lenguaje de la Pontificia Universidad Javeriana (Bogotá), el Programa de Comunicación Social y del Departamento de Humanidades de la Universidad EAFIT (Medellín) y el Núcleo de Estudos da Divulgação Científica del Museu da Vida/Casa de Oswaldo Cruz/Fundação Oswaldo Cruz (Fiocruz) en Rio de Janeiro, Brasil. Cuenta con el apoyo de un convenio de movilidad del Departamento Administrativo de Ciencia, Tecnología e Innovación (COLCIENCIAS) de Colombia y del Conselho Nacional de Desenvolvimento Científico e Tecnológico (CNPq) del Brasil. Está vinculado a la Rede Ibero-Americana de Monitoramento e Capacitação em Jornalismo Científico (www.museudavida.fiocruz.br/redejc). 
2 Durante varios años fue el estudio oficial por la Comisión Nacional de Televisión (CNTV) - hoy transformada en Autoridad Nacional de Televisión (ANTV) - quien estaba encargada de regular dicho medio en Colombia. Durante la época de este artículo (entre abril de 2009 y marzo de 2011), el consumo de tv estuvo cerca del 94\%, como se reporta en: http:/ /www. acimcolombia.com/archivos/FILE_DOC_PUBLICACION/CIFRASEGM-JUNIO2011. pdf. (Fecha de consulta: 27-10-2013).

${ }^{3}$ En Colombia, el término más comúnmente empleado es 'noticiero'; para efectos del presente artículo utilizaremos la palabra 'telediario', para facilitar la comprensión entre lectores de diversos países de habla portuguesa y castellana

${ }^{4}$ La Rede Ibero-americana de Monitoramento e Capacitação em Jornalismo Científico (www. museudavida.fiocruz.br/redejc) ha sido coordinada por el Núcleo de Estudos da Divulgação Científica del Museu da Vida (Rio de Janeiro), que hace parte de la Casa de Oswaldo Cruz/ Fiocruz, en Brasil. Dicha red fue formada en 2009, y está compuesta por investigadores e instituciones de diez países de la región: Argentina, Bolivia, Brasil, Colombia, Cuba, Ecuador, España, México, Portugal y Venezuela. Los autores de este artículo hacen parte de esta red.

${ }^{5}$ Una expresión que a veces se traduce en español como encuadre, pero que no es tan precisa. Para una discusión sobre esto se puede ver Ramalho et al (2012). Al tratar del concepto de encuadres, buscamos identificar los elementos centrales organizadores del mensaje, es decir, los principales enfoques dados por los periodistas a sus noticias (GANS, 1979; GAMSON; MODIGLIANI, 1989).

${ }^{6}$ También en Díaz- del Castillo et al (2012) se usó una parte de este corpus colombiano, que usa elementos similares pero para tratar un tema particular de la ciencia.

${ }^{7}$ En el período estudiado, la aparición de los avances y titulares de los noticieros colombianos no estaba estandarizada y obedecía más bien al vaivén de las lógicas del rating. Por ejemplo, Noticias Caracol suele emitir los titulares de algunas notas en el bloque anterior al que salen emitidas. También hay que tener en cuenta que algunas veces emiten otros avances varios minutos - en ocasiones, decenas de minutos - antes de que empiecen los titulares y la emisión como tal, lo que hace técnicamente difícil establecer lo que hay que incluir en el corpus y su consecución.

${ }^{8}$ Hay que tener presente que las noticias del corpus se desarrollaron en un contexto netamente preelectoral y electoral en Colombia: en marzo de 2010 tuvieron lugar las elecciones parlamentarias y a finales de mayo de 2010 fueron las elecciones presidenciales. Vale la pena anotar que la ciencia no fue objeto de debate en el marco de esta coyuntura noticiosa de elecciones parlamentarias y presidenciales en el corpus de noticias analizado. A esto hay que adicionarle que durante 2009 y hasta febrero de 2010 se mantuvo la incertidumbre por la posibilidad de que el poder ejecutivo y la Corte Constitucional le dieran vía libre a un referendo para cambiar la Constitución para que el ex Presidente Álvaro Uribe se pudiera reelegir para un tercer mandato. Esto copó una parte importante de la agenda de los telediarios, y en particular del de nuestro corpus.

${ }^{9}$ Lo que en otros contextos llaman secciones sobre showbiz. Los telediarios, y más en la época de la muestra, usaban con frecuencia la expresión "las buenas noticias del entretenimiento". Los públicos usan a menudo en Colombia la expresión "los chismes de la farándula". 
${ }^{10}$ En el actual corpus (sin los domingos) hallamos 38 noticias en 72 emisiones revisadas. Sin embargo hubo 6 noticias en total los domingos, lo que en el corpus hubieran representado un 13\% del total del mismo. El corpus total de Colombia habría hecho subir de 38 a 44 el número de notas, en 84 emisiones analizadas, es decir, 0,52 notas por emisión: casi la misma proporción que la del corpus sin los domingos.

${ }^{11}$ Es preciso anotar que esto último cabría para varios aspectos que podrían complementar el análisis de contenido. Uno que, de entrada, es ineludible: la injerencia que puedan llegar a tener los dueños del telediario en su agenda y su contrato de lectura (para usar el concepto de Eliseo Verón, como lo explica De Cheveigné (2000)) con el público. Noticias Caracol pertenece al Canal Caracol, que a su vez pertenece al Grupo Santo Domingo, uno de los grupos económicos más tradicionales y más grandes de Colombia. Dueño, también, desde 1997, del diario El Espectador.

${ }^{12}$ Se trata de dos notas relacionadas con el mismo hecho noticioso: el debate por la caza de un hipopótamo que se escapó del zoológico de la Hacienda Nápoles (que fue construido por Pablo Escobar, el gran capo del narcotráfico, antes de que empezara a ser perseguido de forma continua por las autoridades colombianas). Dicha caza generó un debate entre defensores de los derechos de los animales y autoridades ambientales que argumentaron que era peligroso para los seres humanos y para los hábitats por los que podía deambular.

${ }^{13}$ No fue obvio en varios casos el poder determinar el carácter de investigadores como recurso visual: tuvimos que acudir a otros recursos conexos, ya sea por los créditos que definen a las personas, o por el audio que los señala como 'científicos', por las características del espacio en el que se encuentran, o por lo que ellos mismos dicen, es decir por la información del contexto. En otros casos, tuvimos que acudir a los motores de búsqueda para indagar si la persona que aparece en representación de una institución del conocimiento ha hecho investigación.

${ }^{14}$ Una herramienta de análisis en relación con las imágenes de ciencia, basada en una detallada aproximación semiótica, se puede ver en Sicard (1997).

15 Con base en el protocolo, se incluyó también la variable "localización del científico", con estas posibilidades: laboratorio, oficina, conferencia de prensa, campo, otros. Como esta última da más del $50 \%$, no la tendremos en cuenta en este análisis, pues eso nos indica que para el caso colombiano habría que afinarla más.

${ }^{16}$ En el caso de lo controversial como valor noticioso, el telediario considera como poco noticioso la ciencia y sus polémicas internas. Pareciera que la lógica para abordar controversias por el impacto que la ciencia y la tecnología causan en la sociedad estuviera, en principio, más animada por la relación que existen entre las posturas o decisiones de índole gubernamental/político-normativo que involucran conocimiento científico. Además hay que agregar que de esas 3 notas, 2 están relacionadas con el tema del hipopótamo, mencionado en una nota anterior.

${ }^{17} \mathrm{Al}$ excluir lo implícito de lo científico y al plantear que solo deben aparecer daños, riesgos, promesas o beneficios concretos relacionados con la ciencia y la tecnología, podemos pasar por alto una variedad de mecanismos de construcción de representaciones de la realidad, que en este caso el telediario Noticias Caracol emplea con la ayuda tácita del argumento científico o su valor epistémico de autoridad. Sobre estos elementos tácitos y su fuerza en la construcción de representaciones mediáticas y sociales se puede ver Díaz del Castillo et al (2012).

${ }^{18}$ Es preciso aclarar que se trata de miembros del gobierno de carácter nacional, regional y local. Además hay miembros de otras ramas del Estado (la legislativa y la judicial) que a veces quedan clasificados en esta variable. Pero estos aparecen con poca frecuencia, y no se hace precisión sobre tal diferencia en nuestro protocolo de base en Ramalho et al (2012). 
${ }^{19}$ Ingeominas era el Instituto Colombiano de Investigaciones Geológico-mineras. Hoy en día, la parte geológica la hace el Servicio Geológico Colombiano. IDEAM es el Instituto de Hidrología, Meteorología y Estudios Ambientales de Colombia. Corantioquia es la Corporación Autónoma Regional del Centro de Antioquia, que es responsable de la gestión, protección y promoción de los recursos medioambientales del centro del Departamento de Antioquia.

${ }^{20}$ Los datos oficiales los tiene el sistema de Colciencias, el Departamento Administrativo de Ciencia Tecnología e Innovación. Sin embargo, los datos se basan en su hoja de vida oficial (o CvLac), y hay investigadoras (al igual que investigadores) que, si bien han realizado investigación científica, aún no lo tienen registrado en dicho sistema.

\section{BIBLIOGRAFÍA}

AGUIRRE, J. P. La percepción que tienen los colombianos sobre la ciencia y la tecnología - Encuesta 2004. Bogotá: Colciencias, 2005.

ALMEIDA, C. et al. La cobertura de la ciencia en América Latina: estudio de periódicos de elite en nueve países de la región. In: MORENO, C. (Org.). Periodismo y divulgación cientifica. Tendencias en el ámbito iberoamericano. Madrid: OEI e Biblioteca Nueva, 2011. p. 75-97.

AMORIM, L.; MASSARANI, L. Periodismo científico: un estudio de caso de tres periódicos brasileros. R.B.E.C.T., Ponta grossa, v. 1, n.1, jan/abr, 2008.

ARBOLEDA, T.; HERMELIN, D.; PÉREZ-BUSTOS, T. La cobertura de la ciencia en los noticieros colombianos: del análisis de resultados a las reflexiones metodológicas para su investigación. Revista Ensaio Pesquisa em Educação em Ciências, Rio de Janeiro, v. 14, n. 2, p. 151-166, 2011.

BAUER, M.; RAGNARSDÓTTIR, Á.; RÚDÓLFSDÓTTIR, A. Science and Technology in the British Press, 1946-1990 - A systematics content analysis of the press, work report, 1993.

BECK, U. La sociedad del riesgo. Hacia una nueva modernidad. Barcelona: Paidós, 2006. (Original en alemán: 1986).

BONILLA, J. I.; CADAVID BRINGE, A. ¿Qué es noticia? Agendas, periodistas y ciudadanos. Bogotá: Pontificia Universidad Javeriana - Fundación Konrad Adenauer, 2004.

BONILLA, J. I. et al. De las audiencias contemplativas a los productores conectados: Mapa de los estudios y de las tendencias de los ciudadanos mediáticos. Cali: Pontificia Universidad Javeriana, Medellín: Universidad EAFIT, Bogotá: Universidad de los Andes, 2012.

BUCCHI, M., TRENCH, B. (Eds.) Handbook of public communication of science and technology. Londres: Routledge, 2008.

BUSTOS MORA, G. Ciencia y tecnología en la prensa costarricense: análisis de casos. Revista de Ciencias Sociales, Costa Rica, Universidad de Costa Rica, n. 106-107, p. 215-229, 2004.

DAZA, S.; ARBOLEDA, T. Comunicación pública de la ciencia y la tecnología en Colombia: ¿Políticas para la democratización del conocimiento? Signo y pensamiento, Bogotá, v. 24, n. 50, p. 100-125, 2007.

DE CHEVEIGNÉ, S. L'environnement dans les journaux télévisés. Médiateurs et visions du monde. París: CNRS Éditions, 2000.

DE GREIFF, A.; NIETO, M. Anotaciones para una agenda de investigación sobre las relaciones tecnocientíficas Sur-Norte. Revista de Estudios Sociales, Bogotá, Universidad de los Andes, n. 22, p. 59-69, 2005.

DEMERS, F., LAVIGNE, A. La comunicación pública: una prioridad contemporánea de investigación. Comunicación y sociedad, Guadalajara, Universidad de Guadalajara, n. 18, pp. 65-87, 2007.

DÍAZ DEL CASTILLO, A.; OLARTE SIERRA, M. F.; PÉREZ-BUSTOS, T. Testigos modestos y poblaciones invisibles en la cobertura de la genética humana en los medios de comunicación colombianos. Interface, Comunicação, Saúde, Educaçãoo, Botucatu, v. 16, n. 41, p. 451-467, abr/jun, 2012.

ESCANDELL VIDAL, M. V. Introducción a la pragmática. Barcelona: Editorial Ariel, 1996. 
GAMSON, W.; MODIGLIANI, A. Media discourse and public opinion on nuclear power: A constructionist approach. American Journal of Sociology, [S.1.], v. 95, n. 1, p. 1-7, 1989.

GANS, H. Deciding what's news. New York: Pantheon, 1979.

GONZÁLEZ, M. Del “consejo prematrimonial” al PGH: Treinta años de genética humana en El País (1976-2006), abr. 2008, 384 p., Tesis (Doctorado Interuniversitario en Historia de las Ciencias UB-UAB), Universidad Autónoma de Barcelona, Barcelona, 2008.

GOMEZ-GIRALDO, J. C et al. Los noticieros de la televisión colombiana "en observación": Una mirada desde la academia a la estructura, cobertura y contenidos de los teleinformativos de la televisión abierta en Colombia. Palabra Clave. Bogotá,

v. 13 , n. 2, p. 217-250, 2010.

HERMELIN, D. "Desastres, medios masivos y comunicación pública de la ciencia: entre la vulnerabilidad y la cohesión social en Colombia y América Latina”. Ensaio. Pesquisa em Educaşão em Ciências, ed: Universidad Federal De Minas Gerais, Belo Horizonte, v. 15, n. 3 (en prensa), 2013.

. Un contexto para la comunicación pública de la ciencia y la tecnología en Colombia: de las herencias eurocéntricas a los modelos para la acción. Co-herencia, Medellín, Universidad EAFIT, v. 8, n. 14, p. 231-260, 2011.

. Los estudios de recepción en la formación e investigación sobre periodismo científico en Latinoamérica. In: MASSARANI, L.; RAMALHO, M. (Coord.). Monitoramento e capacitação em jornalismo científico - a experiência de uma rede ibero-americana. Rio de Janeiro: Museu da Vida / Casa de Oswaldo Cruz, Quito: Ciespal, p. 77-96, 2012.

HOLLIMAN, R. Media coverage of cloning: a study of media content, production and reception. Public Understanding of Science, [S.1.], v. 13, n. 2, p. 107-130, 2004.

JACKS, N. Análisis de recepción en América Latina: un recuento histórico con perspectivas al futuro. Quito: CIESPAL, 2011.

KRIPEMDORFF, K. Metodología de análisis de contenido. Teoría y Práctica. Barcelona: Paidós, 1990. (Original en inglés: 1980).

LE BRETON, D. La sociologie du risque. París: Presses Universitares de France (QSJ), 1995.

MAIGRET, E. Sociología de la comunicación y de los medios. Bogotá: F.C.E, 2005. (Original en francés: 2003).

MARTINI, S. Periodismo, noticia y noticiabilidad. Bogotá: Grupo Editorial Norma, 2000.

MASSARANNI, L.; BUYS, B. Cuando la ciencia es noticia: una evaluación de la sección de ciencia en nueve países de América Latina y el Caribe. In: LOZANO, M.; SÁNCHEZ-MORA, C. (Ed.). Evaluando la comunicación de la ciencia: Una perspectiva latinoamericana, México D.F.: CYTED, AECI, DGDC-UNAM, p.115-128, 2008.

MASSARANI, L. et al. A cobertura de ciência por jornais diários: em pauta a pesquisa nacional na Argentina, no Brasil e no México. Rąón y Palabra, México, v. 65, 2008. Disponible en: <http:// www.razonypalabra.org.mx/N/n65/actual/lmassarani.html>. Acceso en: 27 sept. 2012.

MASSARANI, L.; RAMALHO, M. (Coord.). Monitoramento e capacitação em jornalismo científico - a experiência de uma rede ibero-americana. Rio de Janeiro: Museu da Vida / Casa de Oswaldo Cruz, Quito: Ciespal, 2012.

McCOMBS, M. E.; SHAW, D. L. The Evolution of Agenda-Setting Research: Twenty-Five Years in the Marketplace of Ideas. Journal of Communication, [S.1.], v. 43, n. 2, p. 58-67, 1993.

MOIRAND, S. Formes discursives de la diffusion des savoirs dans les médias. Hermès, n. 21, p. $33-$ 44, 1997.

OCyT-COLCIENCIAS. III Encuesta Nacional de Percepción Pública de la Ciencia y la Tecnología. Bogotá: Observatorio Colombiano de Ciencia y Tecnología (OCyT) y Colciencias. Disponible en: http:// 66.7.192.165/ ocytorgc/encuesta/index.php. Acesso en: 30 dic. 2013.

PATIÑO, D. F. Comunicación personal. Director de Noticias Caracol (hasta diciembre de 2011). Entrevista realizada el 29-11-2009. 2009.

PARODI, G., FERRARI, S. El discurso de la ciencia y la tecnología en la prensa escrita chilena: una aproximación al corpus DICIPE-2004. D.E.L.T.A., São Paulo,

v. 23, n. 2, p. 339-369, 2007. 
PELLECHIA, M. G. Trends in science coverage: A content analysis of three US newspapers. Public Understanding of Science, [S.1.], v. 6, n. 1, p. 49-68, 1997.

PÉREZ-BUSTOS, T., LOZANO-BORDA, M. (Eds.). Ciencia, tecnología y democracia. Reflexiones en torno a la apropiación social del conocimiento. Medellín: Colciencias y Universidad EAFIT, 2011.

PETERS, H. P. et al. Interactions with the mass media. Science, EE.UU., v. 321, n. 5886 , p. 204-205, julio, 2008.

RAMALHO, M. et al. Ciência em telejornais: uma proposta de ferramenta para análise de conteúdo de notícias científicas. En: MASSARANI, L.; RAMALHO, M. (Eds.). Monitoramento e capacitação em jornalismo cientifico - a experiência de uma rede ibero-americana. Rio de Janeiro: Museu da Vida / Casa de Oswaldo Cruz / Fiocruz. Quito: Ciespal, 2012.

RAMALHO, M.; POLINO, C.; MASSARANI, L. From the laboratory to prime time: science coverage in the main Brazilian TV newscasts. Journal of Science Communication, [S.l.], v. 11, n. 2, p. 1-11, 2012.

RONDELLI, D.R.R. A ciência no picadeiro: Uma análise das reportagens sobre ciência no programa Fantástico. São Paulo: Umesp. 147p. Dissertação (Mestrado) - Programa de Pós-graduação em Comunicação Social, Universidade Metodista de São Paulo, São Paulo, 2004.

ROZO, C. Representaciones de cultura científica y cultura tecnológica desde los medios de comunicación. Diario El Tiempo, caso de estudio. In: I CONGRESO IBEROAMERICANO DE CIENCIA, TECNOLOGÍA, SOCIEDAD E INNOVACIÓN, 2006, México D.F., Anais..., México OEI, 2006.

SICARD, M. Les paradoxes de l'image. Hermès, Paris, n. 21, p. 45-54, 1997.

THOMPSON, J. The Media and Modernity: A Social Theory of the Media. Cambridge: Polity; Stanford: Stanford University Press, 1995.

Data de recebimento: 30/12/2013

Data de Aprovação: 14/01/2015

Data de Versão Final: 25/02/2015

Contato:

Tania Arboleda

Observatorio Colombiano de ciencia y tecnología.

CARRERA 15 \#37-59 - LA SOLEDAD - BOGOTA - Colômbia - 123456789

Email: taniaarboleda@gmail.com 